\title{
CD44 Standard Isoform; Not a Good Marker for Colon Cancer
}

\author{
Pantea Rohani, ${ }^{1}$ Farahnaz Noroozinia, ${ }^{1}$ Parisa Modarresi, ${ }^{1}$ and Ata Abbasi ${ }^{1,}$ \\ ${ }^{1}$ Department of Pathology, Urmia University of Medical Sciences, Urmia, IR Iran \\ "Corresponding author: Ata Abbasi MD-MPH, APCP, Department of Pathology, Urmia University of Medical Sciences, Urmia, IR Iran. Tel: +98-9124397395, Fax: +98-4433433854, \\ E-mail: aabbasi@alumnus.tums.ac.ir
}

Received 2016 September 27; Accepted 2017 August 30.

\begin{abstract}
Background: CD44 isoforms are known to be possible diagnostic and prognostic markers in colorectal cancer (CRC).

Objectives: The present study aims at investigating the expression of CD44 standard (CD44s) isoform in CRC by immunohistochemistry (IHC) as well as exploring its clinicopathological correlations.

Methods: A total of 102 patients, who underwent surgery for primary colorectal adenocarcinoma, were enrolled in this study. IHC staining for CD44 was performed on pathology samples and defined as: staining $<10 \%$ as low/weak, $10 \%-50 \%$ as moderate, and $>$ $50 \%$ as strong/extensive expression level.

Results: The patients' mean age was $62.8 \pm 15.67$ years. IHC staining for CD44s demonstrated low expression level in 56\%, moderate in $24 \%$, and extensive in $20 \%$ of the participants. The only association was found between CD44 expression level and patients' age (P $=0.03$ ) as $25 \%$ of tumors in patients younger than 60 years old expressed CD44 expression strongly in contrast to $16.1 \%$ of the patients older than 60 years old. No other association was found between tumor histology, stage, gender, tumor location, lymphovascular invasion, perineural invasion, lymph node metastasis, or tumor subtype $(\mathrm{P}>0.05)$.

Conclusions: No relation was found between CD44s expression and any tumoral characteristics of CRC. It seems that CD44s are not helpful in improving the prognostic or diagnostic power in CRC.
\end{abstract}

Keywords: CD44, Isoform, Colorectal Cancer

\section{Background}

Colorectal cancer is one of the most frequent neoplastic diseases in human, ranked after lung and prostatic cancers in men and breast, lung, and cervical cancers in women (1). In developed countries, colorectal cancer (CRC), or rather its progression to metastatic disease, accounts for $25 \%$ of tumor deaths (2). CRC evolves through a series of morphologically recognizable stages, known as the adenoma-carcinoma sequence. Currently, predicting patients' prognosis is mainly based on the stage of CRC at the time of resection, histopathological grade of tumor, and changes in serum CEA levels $(3,4)$. However, clinical outcomes of patients with CRC is still difficult to predict; therefore, additional prognostic markers are required (3, $5,6)$.

CD44 was, first, identified as cell surface hyaluronan receptor, but it is, now, known to be expressed in colonic mucosa and present in many physiologic and pathologic processes, including cell division, migration and adhesion, and survival $(3,7,8)$. CD44 gene has almost 10 variable exons, leading to various patterns of splicing; that is how CD44 has multiple isoforms rather than its standard form
(8). Some particular CD44 isoforms, including standard form (CD44s), V6, and V9 isoforms had a correlation with the depth of invasion, lymph node involvement, and overall survival in CRC $(1,3,6$, 9-15). However, some controversies could be observed in some studies with completely different results $(7,16-20)$.

The present study aims at investigating the expression of CD44s in primary CRC by immunohistochemistry (IHC) as well as exploring its relation with clinicopathological characteristics.

\section{Methods}

\subsection{Patients}

A total of 102 patients were enrolled in this study. They had undergone surgical resection for primary colorectal adenocarcinoma at the department of surgery, Urmia Imam Khomeini hospital, Urmia, Iran between 2010 and 2012. Tumor staging was based on American Joint Committee on Cancer (AJCC) and Union Inrernational Contre le Cancer (UICC) systems (21). Tumors were histologically classified as well differentiated, moderately differen- 
tiated, and poorly differentiated adenocarcinoma according to WHO classification (22). The study is reviewed and approved by ethics committee of Urmia University of Medical Sciences. As the study was performed on paraffin blocks no written informed consent was obtained from any of the patients.

\subsection{Tissue Specimens and Immunohistochemistry}

The non-necrotic portion of the tumors and surrounding normal mucosa were processed, using the standard paraffin wax technique after fixation in $10 \%$ formalin for 24 hours, and sections were stained with hematoxylin and eosin (H\&E). From representative blocks of each case, $4 \mu \mathrm{m}$ thick sections were obtained for IHC staining. IHC staining for CD44 was performed according to manufacturer's protocol (clone DF 1485, DAKO Corporation, Glostrup, Denmark, mouse type). In brief, sections were deparaffinized in xylene for 30 minutes and rehydrated with graded alcohols and subjected to Tris-EDTA. After antigen retrieval, the slides were rinsed in TBS and incubated to quench endogenous peroxidase activity with $3 \%$ hydrogen peroxide for 5 minutes. In order to reduce non-specific binding of antisera, sections were washed with TBS (15 minutes) before application of the primary anti-CD44 antibody (clone DF 1485, Dako Denmark, mouse type) for 1 hour in room temperature. Then, sections were rinsed in TBS again and were, subsequently, treated with envision-plus (DAKO Corporation, Glostrup, Denmark) for 30 minutes. The reactions were visualized with diaminobenzidine (DAB) as a chromogen. Finally, sections were counterstained with hematoxylin, dehydrated, and mounted. Tumor cells with cytoplasmic or membranous staining pattern were regarded as positive. Staining less than $10 \%$ were defined as low/weak, $10 \%$ - 50\% as moderate, and $>50 \%$ as strong/extensive expression. The staining of normal colonic mucosa, predominantly, in basal region of crypts was considered as internal positive control.

\subsection{Statistical Analysis}

The results are expressed as mean $\pm S D$. Statistical analysis was performed, using SPSS version 16.0.1 (SPSS Inc., Chicago, IL, U.S.A.). The statistical differences between proportions were determined by $\chi^{2}$ analysis. Numerical data were evaluated, the using analysis of variance, followed by Tukey's post hoc test. $\mathrm{P}<0.05$ was considered as significant.

\section{Results}

Among the patients, 57 were men and 45 were women with the mean age of $62.8 \pm 15.67$ years $(29-100)$ and the

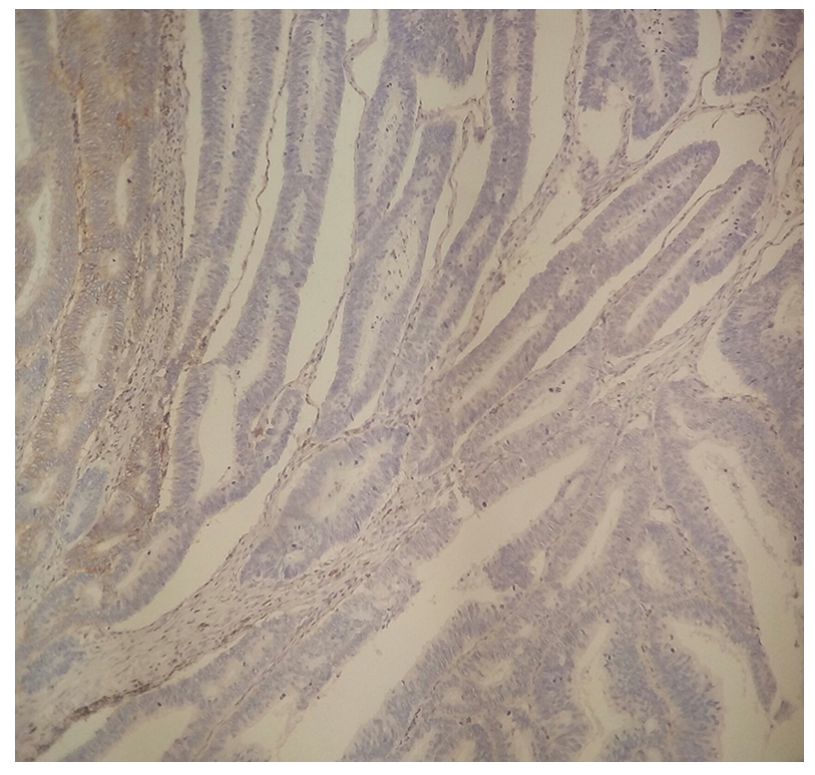

Figure 1. IHC Staining Showing Low Expression of CD44 ( $<10 \%$ of Tumoral Cells are Stained by CD44), (20x)

mean tumor size of $5.61 \pm 3.75 \mathrm{~cm} \mathrm{(1.5} \mathrm{-} \mathrm{30).} \mathrm{IHC} \mathrm{stain-}$ ing for CD44 demonstrated low expression level $(<10 \%$ of neoplastic cells) in $56 \%$, moderate $(10 \%-50 \%$ of neoplastic cells) in $24 \%$, and extensive in $20 \%$ of the cases (Figures 1 and 2).

The patients' demographic data and relationship between CD44 expression and clinicopathologic parameters are shown in Table 1. No association was found between CD44 expression and tumor histology $(\mathrm{P}=0.982)$, tumor stage $(\mathrm{P}=0.695)$, gender $(\mathrm{P}=0.054)$, tumor location $(\mathrm{P}=$ $0.830)$, lymphovascular invasion $(\mathrm{P}=0.318)$, perineural invasion $(\mathrm{P}=0.538)$, lymph node metastasis $(\mathrm{P}=0.705)$, or tumor subtype (non-mucinous versus mucinous adenocarcinoma $)(P=0.510)$. The only association was found between $\mathrm{CD} 44$ expression level and patients' age $(\mathrm{p}=0.03)$. Twentyfive percent of tumors in patients younger than 60 years expressed CD44 expression strongly, whereas $16.1 \%$ of patients with 60 years or older had strong CD44 expression (Table 1).

\section{Discussion}

CD44 complex forms a family of cell surface adhesion molecules, which are involved in cell-cell and cellmatrix interactions, lymphocyte activation and homing, cell motility, differentiation, and migration $(8,23)$. The multiple protein isoforms are encoded by alternative splicing of a single gene and are, further, modified by a range of post-translational modifications. 
Table 1. Characteristics of Participants and Their Associations with CD44 Expression

\begin{tabular}{|c|c|c|c|c|c|c|}
\hline CD44 Expression level & & & Low & Moderate & Strong & \\
\hline \multirow{3}{*}{ Stage } & I & 9 & $4(44.4)$ & $3(33.3)$ & $2(22.2)$ & \multirow{3}{*}{0.695} \\
\hline & II & 55 & $29(52.7)$ & $14(25.4)$ & $12(21.9)$ & \\
\hline & III, IV & 38 & $25(65.8)$ & $7(18.4)$ & $6(15.8)$ & \\
\hline \multirow{2}{*}{ Grade(Differentiation) } & Well & 69 & $38(55)$ & $17(24.6)$ & $14(20.2)$ & \multirow{2}{*}{0.982} \\
\hline & Poor & 7 & $5(71.4)$ & $1(14.3)$ & $1(14.3)$ & \\
\hline \multirow{2}{*}{ Age } & $<60$ & 45 & $29(64.4)$ & $5(11.1)$ & $11(24.5)$ & \multirow{2}{*}{$0.03^{\mathrm{a}}$} \\
\hline & $\geq 60$ & 57 & $29(50.8)$ & $19(33.3)$ & $9(15.9)$ & \\
\hline \multirow{2}{*}{ Gender } & Male & 57 & $34(59.6)$ & $14(24.5)$ & $9(15.9)$ & \multirow{2}{*}{0.054} \\
\hline & Female & 45 & $24(53.3)$ & $10(22.3)$ & $11(24.4)$ & \\
\hline \multirow{2}{*}{ Tumor location } & Colon & 81 & $44(54.3)$ & $20(24.7)$ & $17(21)$ & \multirow{2}{*}{0.83} \\
\hline & Rectum & 21 & $13(61.9)$ & $5(23.8)$ & $3(14.3)$ & \\
\hline \multirow{2}{*}{ Vascular invasion } & NO & 56 & $30(53.5)$ & $12(21.4)$ & $14(25.1)$ & \multirow{2}{*}{0.318} \\
\hline & YES & 46 & $28(60.8)$ & $12(26.2)$ & $6(13)$ & \\
\hline \multirow{2}{*}{ Lymph node metastasis } & NO & 67 & $36(53.7)$ & $17(25.3)$ & $14(21)$ & \multirow{2}{*}{0.705} \\
\hline & YES & 35 & $22(62.8)$ & $7(20.1)$ & $6(17.1)$ & \\
\hline \multirow{2}{*}{ Tumor size, $\mathbf{c m}$} & $<5$ & 50 & $29(58)$ & $13(26)$ & $8(16)$ & \multirow{2}{*}{0.629} \\
\hline & $\geq 5$ & 52 & $29(55.7)$ & $11(21.2)$ & $12(23.1)$ & \\
\hline
\end{tabular}

${ }^{\mathrm{a}}$ Significant.

In the present study, we used immunohistochemical staining against CD44s (standard) isoform. In this study, it was shown that CD44s expression was not correlated with clinicopathological characteristics of CRC, including tumor grade, the depth of invasion, and vascular/perineural invasion. Although some studies have reported that the decreased CD44 expression is an independent predictor of nodal metastasis $(7,17)$, no association, in this study, was observed between CD44 expression and nodal metastasis.

In contrast, Zavrides et al. reported that CD44 expression was associated with pathologic stage, histologic grade, and tumor location (1).

Asao et al. demonstrated that CD44 expression had decreased regional lymph node metastasis in colorectal cancers. They explained that this suppression activity may be due to CD44 binding to extracellular matrix in the submucosal layer, immobilizing cancer cells, and preventing their spread (18).

In the same vein, Huh et al. reported that CD44 overexpression was correlated with the depth of invasion, lymphocyte involvement, and it may be an independent unfavorable prognostic factor for overall survival in advanced CRC, especially in stage IV (9).
Thus, there are still controversies on the definite role of CD44 and its isoforms in cancer development and progression. These discrepancies may be due to different patient material and follow up duration, antibodies, and immunohistochemical techniques used in different studies $(2,9)$.

Furthermore, a number of studies have demonstrated CD44 expression in most non-tumoral epithelial tissues, including stomach mucous membrane, small intestinal, prostate, ductal epithelium of breast, skin, hair follicles, and transitional epithelium $(1,2,9)$. Likewise, CD44 expression is found in normal colonic crypt epithelium, predominantly in basal region of crypts, and benign neoplastic tissues, such as adenomatous polyps $(16,24,25)$. Such results argue the role of CD44 as an indicator of cancerous process.

Herlich et al. reported that CD44 could act as both tumor promoter and tumor suppressor molecule, depending on its site of action in cancerous cells. This interesting finding would be another explanation for discrepancies and controversies in the literature about the role of CD44 in cancer (26).

CD44 can cause complexities with various molecules, including some growth factors such as EGFR receptors and 
HER2 as well as activating them; through this mechanism, CD44 can act as a tumor promoter molecule. Hepatocyte growth factor (HGF) is another growth factor (GF), which can be modulated by CD44 and is one of the important GFs involved in colorectal cancer development (8). P53, TGF$\mathrm{b}$, and matrix metaloproteinases (MMPs) are other growth factors that can be modulated by CD44 and can be involved in CD44 induced tumorigenesis (8). Other studies have reported tumor suppressing activity of CD44 (27). It has been explained that dual activity of CD44 is related to its molecular weight, in which high molecular weight CD44 variants are tumor suppressors, and low molecular weights may act as tumor promoters (8).

Totally, no relation was found between CD44s expression and any tumoral characteristics of colorectal cancer. Considering the controversies in CD44 mechanism of action, further studies are suggested in different variants of this molecule so as to make its role more understandable in colon cancers.

\section{Acknowledgments}

This study is supported by research funds of Urmia University of Medical Sciences. We would like to thank Mr. Ja-

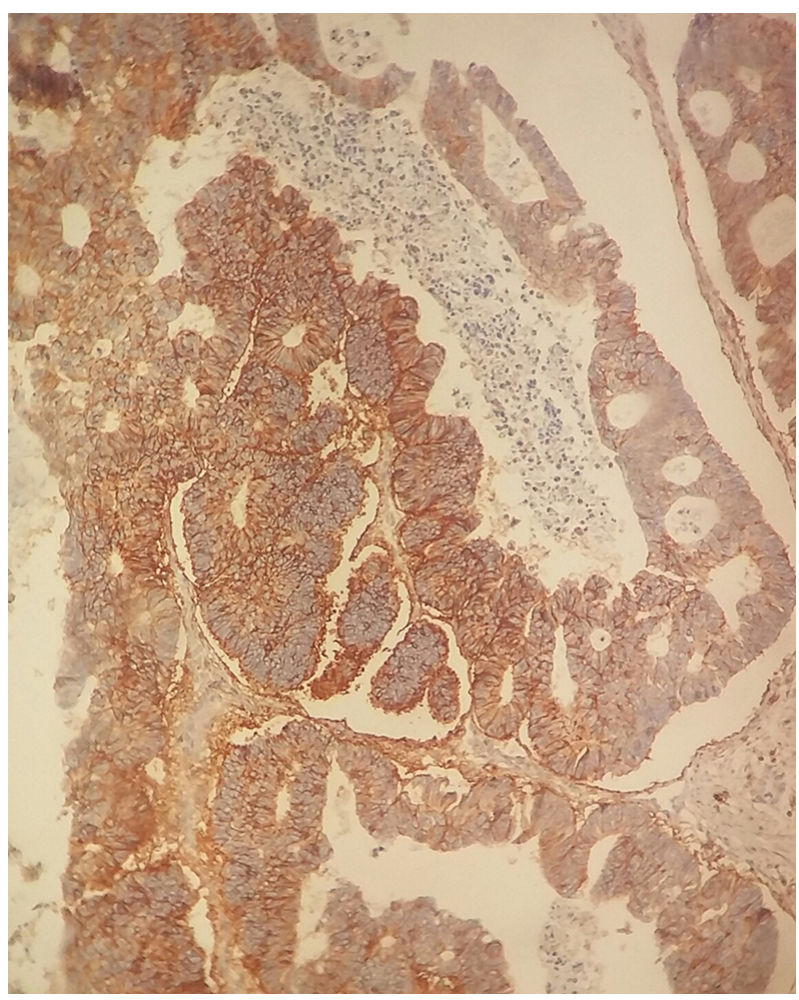

Figure 2. IHC Staining for CD44 with Strong Positivity (20x) fari for his technical support.

\section{Footnotes}

Authors' Contribution: None declared.

Financial Disclosure: None declared.

Conflict of Interest: None declared.s

\section{References}

1. Zavrides HN, Zizi-Sermpetzoglou A, Panousopoulos D, Athanasas G, Elemenoglou I, Peros G. Prognostic evaluation of CD44 expression in correlation with bcl-2 and p53 in colorectal cancer. Folia Histochem Cytobiol. 2005;43(1):31-6. [PubMed: 15871560].

2. Banky B, Raso-Barnett L, Barbai T, Timar J, Becsagh P, Raso E. Characteristics of CD44 alternative splice pattern in the course of human colorectal adenocarcinoma progression. Mol Cancer. 2012;11:83. doi: 10.1186/1476-4598-11-83. [PubMed: 23151220].

3. Mulder JW, Wielenga VJ, Polak MM, Van den Berg FM, Adolf GR, Herrlich $\mathrm{P}$, et al. Expression of mutant p53 protein and CD44 variant proteins in colorectal tumorogenesis. GUT. 1994;36:76-80.

4. Zalewski B. Levels of v5 and v6 CD44 splice variants in serum of patients with colorectal cancer are not correlated with pT stage, histopathological grade of malignancy and clinical features. World J Gastroenterol. 2004;10(4):583-5. [PubMed:14966921].

5. Furuta K, Zahurak M, Goodman SN, Hamilton SR, August JT. CD44 expression in the stromal matrix of colorectal cancer: association with prognosis. Clin Cancer Res. 1998;4(1):21-9. [PubMed: 9516948].

6. Kimura Y, Goi T, Nakazawa T, Hirono Y, Katayama K, Urano T, et al. CD44variant exon 9 plays an important role in colon cancer initiating cells. Oncotarget. 2013;4(5):785-91. doi: 10.18632/oncotarget.1048. [PubMed: 23800986].

7. Kunimura T, Yoshida T, Sugiyama T, Morohoshi T. The Relationships Between Loss of Standard CD44 Expression and Lymph Node, Liver Metastasis in T3 Colorectal Carcinoma. J Gastrointest Cancer. 2009;40(3-4):115-8. doi: 10.1007/s12029-009-9100-0. [PubMed: 19937401].

8. Inoue K, Fry EA. Aberrant Splicing of Estrogen Receptor, HER2, and CD44 Genes in Breast Cancer. Genet Epigenet. 2015;7:19-32. doi: 10.4137/GEG.S35500. [PubMed: 26692764].

9. Huh JW, Kim HR, Kim YJ, Lee JH, Park YS, Cho SH, et al. Expression of standard CD44 in human colorectal carcinoma: association with prognosis. Pathol Int. 2009;59(4):241-6. doi: 10.1111/j.14401827.2009.02357.x. [PubMed: 19351367].

10. Liu YJ, Yan PS, Li J, Jia JF. Expression and significance of CD44s, CD44v6, and nm23 mRNA in human cancer. World J Gastroenterol. 2005;11(42):6601-6. [PubMed: 16425351].

11. Bhatavdekar JM, Patel DD, Chikhlikar PR, Trivedi TI, Gosalia NM, Ghosh $\mathrm{N}$, et al. Overexpression of CD44: A useful independent predictor of prognosis in patients with colorectal carcinomas. Ann Surg Oncol. 1998;5(6):495-501.

12. Saigusa S, Inoue $Y$, Tanaka K, Toiyama Y, Matsushita K, Kawamura M et al. Clinical significance of LGR5 and CD44 expression in locally advanced rectal cancer after preoperative chemoradiotherapy. Int J Oncol. 2012;41(5):1643-52. doi: 10.3892/ijo.2012.1598. [PubMed: 22923071].

13. Wan XB, Pan ZZ, Ren YK, Ding PR, Chen G, Wan DS. Expression and clinical significance of metastasis-related tumor markers in colorectal cancer. Chin J Cancer. 2009;28(9):1-6.

14. Nihei Z, Ichikawa W, Kojima K, Togo S, Miyanaga T, Hirayama R, et al. The positive relationship between the expression of CD44 variant 6 and prognosis in colorectal cancer. Surg Today. 1996;26(9):760-1. [PubMed: 8883258]. 
15. Garouniatis A, Zizi-Sermpetzoglou A, Rizos S, Kostakis A, Nikiteas N, Papavassiliou AG. FAK, CD44v6, c-Met and EGFR in colorectal cancer parameters: tumour progression, metastasis, patient survival and receptor crosstalk. Int J Colorectal Dis. 2013;28(1):9-18. doi: 10.1007/s00384-012-1520-9. [PubMed: 22733437].

16. Jackson PA, Green MA, Pouli A, Hubbard R, Marks CG, Cook MG. Relation between stage, grade, proliferation, and expression of p53 and CD44 in adenomas and carcinomas of the colorectum. J Clin Pathol. 1995;48(12):1098-101. [PubMed: 8567994].

17. Al-Maghrabi J, Gomaa W, Buhmeida A, Al-Qahtani M, Al-Ahwal M. Decreased immunoexpression of standard form of CD44 is an independent favourable predictor of nodal metastasis in colorectal carcinoma. Anticancer Res. 2012;32(8):3455-61. [PubMed: 22843930].

18. Asao T, Nakamura J, Shitara Y, Tsutsumi S, Mochiki E, Shimura T, et al. Loss of standard type of CD44 expression in invaded area as a good indicator of lymph-node metastasis in colorectal carcinoma. Dis Colon Rectum. 2000;43(9):1250-4. [PubMed: 11005492] discussion 1254-5.

19. Avoranta ST, Korkeila EA, Syrjanen KJ, Pyrhonen SO, Sundstrom JT. Lack of CD44 variant 6 expression in rectal cancer invasive front associates with early recurrence. World J Gastroenterol. 2012;18(33):454956. doi:10.3748/wjg.v18.i33.4549. [PubMed: 22969228].

20. Li XD, Ji M, Wu J, Jiang JT, Wu CP. Clinical significance of CD44 variants expression in colorectal cancer. Tumori. 2013;99(1):88-92. doi
10.1700/1248.13794. [PubMed: 23549006].

21. Rosai J, Ackerman LV. Rosai and Ackerman's surgical pathology. 9th ed. Edinburgh ; New York : Mosby; 2011.

22. World Health Organization. Histological typing of intestinal tumors. Geneva: WHO; 1976 .

23. Goodison S, Urquidi V, Tarin D. CD44 cell adhesion molecules. Mol Pathol. 1999;52(4):189-96. [PubMed: 10694938].

24. Fox SB, Fawcett J, Jackson DG, Collins I, Gatter KC, Harris AL, et al. Normal human tissues, in addition to some tumors, express multiple different CD44 isoforms. Cancer Res. 1994;54(16):4539-46. [PubMed: 7519124].

25. Gotley DC, Fawcett J, Walsh MD, Reeder JA, Simmons DL, Antalis TM. Alternatively spliced variants of the cell adhesion molecule CD44 and tumour progression in colorectal cancer. Br J Cancer. 1996;74(3):34251. [PubMed: 8695347].

26. Herrlich P, Morrison H, Sleeman J, Orian-Rousseau V, Konig H, WegRemers S, et al. CD44 acts both as a growth- and invasivenesspromoting molecule and as a tumor-suppressing cofactor. Ann N Y Acad Sci. 2000;910:106-18. [PubMed: 10911909] discussion 118-20.

27. Louderbough JM, Schroeder JA. Understanding the dual nature of CD44 in breast cancer progression. Mol Cancer Res. 2011;9(12):1573-86. doi: 10.1158/1541-7786.MCR-11-0156. [PubMed: 21970856]. 